\title{
Drell-Yan lepton angular distributions in perturbative QCD
}

\author{
Martin Lambertsen* \\ Tübingen University \\ E-mail: martin.lambertsen@uni-tuebingen.de \\ Werner Vogelsang ${ }^{\dagger}$ \\ Tübingen University \\ E-mail: werner.vogelsang@uni-tuebingen.de
}

\begin{abstract}
We present a comprehensive comparison of the available experimental data for the Drell-Yan lepton angular coefficients $\lambda$ and $v$ to calculations at leading and next-to-leading order (NLO) of perturbative QCD. To obtain the NLO corrections, we make use of publicly available numerical codes that allow us to compute the Drell-Yan cross section at second order in perturbation theory and from which the contributions we need can be extracted. Our comparisons show that overall perturbative QCD is able to describe the experimental data rather well, even in the fixed-target regime. There appears to be little evidence for effects that go beyond fixed-order collinear factorized perturbation theory, although the presence of such effects is not ruled out. We also address the recent ATLAS data which show tension with NLO theory for the coefficient $v$.
\end{abstract}

QCD Evolution 2016

May 30 - June 03, 2016

National Institute for Subatomic Physics (Nikhef), Amsterdam

\footnotetext{
*The author acknowledges support by the state of Baden-Württemberg through bwHPC.

${ }^{\dagger}$ Speaker.

${ }^{\ddagger}$ This work was supported in by the Deutsche Forschungsgemeinschaft (Grant No. VO 1049/1).
} 


\section{Introduction}

It has been known for a long time $[1,2]$ that leptons produced in the Drell-Yan process $H_{1} H_{2} \rightarrow$ $\ell \bar{\ell} X$ may show nontrivial angular distributions. We denote the momentum of the intermediate virtual boson $V=\gamma^{*}, Z$ that decays into the lepton pair by $q$. In a specific rest frame of the virtual boson (for our purposes, the Collins-Soper frame [1]) we can define polar and azimuthal lepton decay angles $\theta$ and $\phi$, respectively. Considering, for simplicity, a situation where contributions by $Z$-bosons are negligible and only the exchange of an intermediate virtual photon is relevant, one can show that the cross section differential in $d^{4} q$ and $d \Omega \equiv d \cos \theta d \phi$ may be written as

$$
\frac{d \sigma}{d^{4} q d \Omega}=\frac{\alpha^{2}}{2 \pi N_{c} Q^{2} s^{2}}\left(W_{T}\left(1+\cos ^{2} \theta\right)+W_{L}\left(1-\cos ^{2} \theta\right)+W_{\Delta} \sin 2 \theta \cos \phi+W_{\Delta \Delta} \sin ^{2} \theta \cos 2 \phi\right),
$$

where $\alpha$ is the fine structure constant, $N_{c}=3$ the number of colors in QCD, $Q^{2}=q^{2}$ and $s$ the c.m.s. energy squared of the incoming hadrons $H_{1}$ and $H_{2}$. The structure functions $W_{T}, W_{L}, W_{\Delta}$, $W_{\Delta \Delta}$ are functions of $q$. If also $Z$-bosons contribute, there are additional angular terms and structure functions in the cross section formula. For details of the derivation of the cross section (also for discussion of other related reference frames), see Refs. [1, 2, 3, 4, 5].

From the differential cross section one easily derives an expression for the normalized decay angle distribution

$$
\frac{d N}{d \Omega} \equiv\left(\frac{d \sigma}{d^{4} q}\right)^{-1} \frac{d \sigma}{d \Omega d^{4} q}
$$

One usually writes this as

$$
\frac{d N}{d \Omega}=\frac{3}{4 \pi} \frac{1}{\lambda+3}\left[1+\lambda \cos ^{2} \theta+\mu \sin 2 \theta \cos \phi+\frac{v}{2} \sin ^{2} \theta \cos 2 \phi\right],
$$

where

$$
\lambda=\frac{W_{T}-W_{L}}{W_{T}+W_{L}}, \quad \mu=\frac{W_{\Delta}}{W_{T}+W_{L}}, \quad v=\frac{2 W_{\Delta \Delta}}{W_{T}+W_{L}} .
$$

Much effort has gone into studies of these angular coefficients $\lambda, \mu, v$, both experimentally and theoretically. On the experimental side, measurements of the coefficients are by now available over a wide range of kinematics, from fixed-target energies $[6,7,8,9]$ all the way to the Tevatron [10] $p \bar{p}$ and the LHC $p p$ colliders $[11,12]$. In the fixed-target regime various combinations of beams and targets are available; data have been taken with pion beams off nuclear (tungsten) targets [6,7] and also for $p p$ and $p d$ collisions $[8,9]$. The experimental results are typically given as functions of the transverse momentum $q_{T}$ of the virtual boson, in a certain range of the lepton pair mass, $Q \equiv \sqrt{Q^{2}}$. For the fixed-target data, $q_{T}$ is limited to a few $\mathrm{GeV}$ and $Q$ is usually around $5-10 \mathrm{GeV}$. This is very different for the high-energy collider measurements which are carried out around $Q=m_{Z}$, where $m_{Z}$ is the $Z$-boson mass. The range in $q_{T}$ explored here is much larger and reaches to almost $100 \mathrm{GeV}$ at the Tevatron and even much beyond that at the LHC.

The lowest-order (LO) partonic channel $q \bar{q} \rightarrow V(\rightarrow \ell \bar{\ell})$ with collinear incoming partons leads to the prediction $\lambda=1, \mu=v=0$. However, for this process the virtual photon has vanishing transverse momentum, $q_{T}=0$, so it cannot contribute to the cross section at finite $q_{T}$. The situation changes when "intrinsic" parton transverse momenta are taken into account. The coefficient 
$v$, especially, which corresponds to a $\cos 2 \phi$ dependence in azimuthal angle, has received a lot of attention in this context since it was discovered [13] that it may probe interesting novel parton distribution functions of the nucleon, known as Boer-Mulders functions [14]. These functions represent a transverse-polarization asymmetry of quarks inside an unpolarized hadron and are "T-odd" and hence related to nontrivial (re)scattering effects in QCD (see [15]). Detailed phenomenological $[16,17]$ or model-based [18] studies have been presented that confront the fixed-target experimental data with theoretical expectations based on the Boer-Mulders functions.

Already the early theoretical studies $[19,20,21,22,23]$ revealed that also plain perturbativeQCD radiative effects lead to departures from the simple prediction $\lambda=1, \mu=v=0$, starting from $\mathscr{O}\left(\alpha_{s}\right)$ with the processes $q \bar{q} \rightarrow V g$ and $q g \rightarrow V q$. At $q_{T} \neq 0$ in fact the latter processes become the LO ones. A venerable result of $[2,24]$ obtained on the basis of these LO reactions is the Lam-Tung relation,

$$
1-\lambda-2 v=0
$$

which holds separately for both partonic channels in the Collins-Soper frame [1]. Next-to-leading order (NLO) corrections to the cross sections relevant for the angular coefficients have first been derived in Refs. [25, 26]. These suggest overall modest $\mathscr{O}\left(\alpha_{s}^{2}\right)$ effects on $\lambda, \mu, v$, so that also the Lam-Tung relation, although found to be violated at NLO, still holds to fairly good approximation. The data from the fixed-target experiment E615 [6] indicate a violation of the Lam-Tung relation, while the other fixed-target sets are overall consistent with it, as are the Tevatron data [10]. A clear violation of the Lam-Tung relation, on the other hand, was observed recently at the highest energies, in $p p$ collisions at the LHC $[11,12]$.

In [27], we took a fresh look at the Drell-Yan angular dependences in the framework of perturbative QCD. Specifically, we presented an exhaustive comparison of the LO and NLO QCD predictions for the parameters $\lambda$ and $v$ with the experimental data, over the whole energy range available. Rather than attempting to retrieve the results of $[25,26]$, we determined new NLO predictions. For this purpose, we used the publicly available codes FEWZ (version 3.1) [28] and DYNNLO [29]. The details of the computation concerning the extraction of the angular coefficients and the choices of parton distribution functions and scales can be found in [27]. Our study was very much inspired by the recent work [30], in which the authors pointed out that the most likely physical explanation for the CMS result [11] on the violation of the Lam-Tung relation is QCD radiative effects at NLO. We indeed confirmed this in our study. New results from ATLAS [12], on the other hand, show tension with the theoretical results even at NLO, as will be discussed below.

Although clearly collinear perturbation theory at fixed-order (NLO) cannot provide a completely adequate framework for describing cross sections in all kinematic regimes of interest for the angular coefficients, our results to be presented below yield important benchmarks. In our view, it appears likely that fixed-order perturbation theory will work much better for ratios of cross sections than for the cross sections themselves. In fact, we can describe most data sets well and do not find any clear-cut evidence for nontrivial additional contributions to be attributed to parton intrinsic momenta. We stress that QCD radiative effects are typically not considered at all when for example Boer-Mulders functions are extracted from data for $v$ (although the conceptual framework for such a combined analysis is available [31]). At the very least, our results establish the relevance of the radiative effects for phenomenological studies of the Drell-Yan angular dependences. 


\section{Comparison to data}

We now present comparisons of the theoretical predictions at LO and NLO to the available experimental data for the angular coefficients $\lambda$ and $v$. We do not show any results for the coefficient $\mu$ which comes out always extremely small and in fact usually consistent with zero both in the theoretical calculation and in experiment, within the respective uncertainties. The results are essentially presented in the order of decreasing energy, starting with a comparison to the highenergy CMS data from the LHC [11] (we will, however, address the ATLAS data only at the end of our paper) and Tevatron [10]. For these data sets $Q$ is very large, $Q \sim m_{Z}$, so that perturbative methods should be well justified. The transverse momentum $q_{T}$ varies over a broad range, taking low values as well as values of order $Q$. At the lower end, where $q_{T} \ll Q$, it may well be necessary to perform an all-order resummation of perturbative double logarithms in $q_{T} / Q$ in order to describe the Drell-Yan cross section properly. However, such logarithms are expected to cancel to a large extent in the angular coefficients [3,4]. Thus, if ever fixed-order perturbative QCD predictions are able to provide an adequate description of the angular coefficients, it should be in the kinematic regimes explored at the LHC and Tevatron.

Figures $1 \mathrm{a}$ and $1 \mathrm{~b}$ show our results for $\lambda$ and $v$ compared to the CMS data [11], for two separate bins in the rapidity of the virtual boson $\eta \equiv \log \left(\left(q_{0}+q_{L}\right) /\left(q_{0}-q_{L}\right)\right) / 2$. We note that CMS presents their data in terms of a different set of angular coefficients termed $A_{0}, A_{1}, A_{2}, A_{3}$, which are directly related to the coefficients we use here. In particular, we have $\lambda=\left(2-3 A_{0}\right) /\left(2+A_{0}\right)$ and $v=2 A_{2} /\left(2+A_{0}\right)$. As in Ref. [30], in order to present a full comparison in terms of $\lambda$ and $v$, we transform the experimental data correspondingly. Here we have propagated the experimental uncertainties, albeit without taking into account any correlations. The lines in the figures show our LO results for the coefficients. As one can see, they qualitatively follow the trend of the data, but for the coefficient $v$ a clear deviation between data and LO theory is observed. This is precisely the finding also emphasized in Ref. [30] where it was argued (without explicit NLO calculation) that the discrepancy ought to be related to higher-order QCD effects. Indeed, this is what we find. The NLO results (histograms) show a markedly better agreement with the data, which in fact is nearly perfect. The coefficient $\lambda$, on the other hand, changes only marginally from LO to NLO. As is visible in the figures, the results at very high values of $q_{T}$ are numerically less accurate, as shown by the somewhat erratic behavior of the histograms. In order to collect higher statistics, we have also performed runs for which we integrated over only eight $q_{T}$ bins, choosing exactly the ones used in the experimental analysis. The corresponding results are shown in Fig. 1c for the range $1<|\eta|<2.1$. Our goal was to make sure that the numerical uncertainty for these bins is much smaller than the experimental one even in the bin at highest $q_{T}$. The figure once more impressively shows how NLO theory leads to an excellent description of the CMS data.

It is interesting to note that NLO FEWZ results were also shown in the CMS paper [11]. However, the agreement with the data for the coefficient $A_{2}$ (which multiplies the $\cos 2 \phi$ dependence of the cross section) reported there appears to be not quite as good as the one we find for our coefficient $v$. It is conceivable that our computation of the coefficients is numerically more stable.

We next turn to the comparison to the CDF data [10] taken in $p \bar{p}$ collisions at $\sqrt{s}=1960 \mathrm{GeV}$ at the Tevatron. The results are shown in Fig. 1d. We observe that both the LO and the NLO results are in good agreement with the data, NLO doing a bit better overall. Both coefficients $\lambda$ and $v$ 

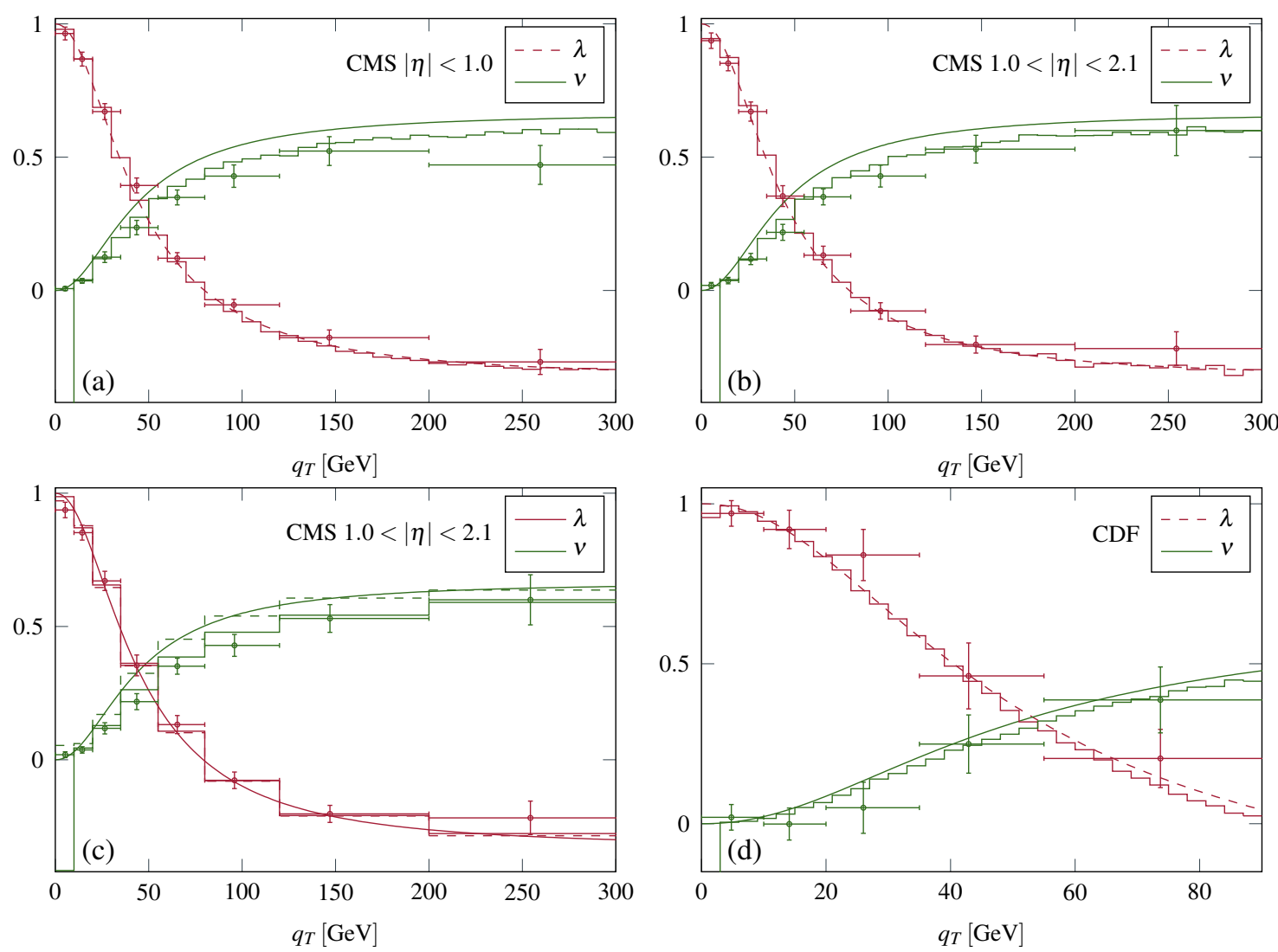

Figure 1: Comparison of LO (lines) and NLO (FEWZ [28], histograms) theoretical results for the angular coefficients $\lambda$ and $v$ to CMS [11] ((a), (b) and (b)) and CDF [10] (d) data. CMS data are taken at $\sqrt{s}=8 \mathrm{TeV}$, integrated over $81 \leq Q \leq 101 \mathrm{GeV}$ and over a midrapidity interval $|\eta|<1$ and a forward/backward rapidity interval $1<|\eta|<2.1$ of the virtual boson, respectively. (c) is the same as (b), but with the NLO theoretical results integrated over the eight $q_{T}$ bins used by CMS. In this figure, the dashed histograms show the LO results and the solid ones the NLO results. To guide the eye, we also show the LO results as smooth lines. CDF data are taken in $p \bar{p}$ scattering at $\sqrt{s}=1960 \mathrm{GeV}$ integrated over $66 \leq Q \leq 116 \mathrm{GeV}$ and over $|\eta|<3.6$ of the virtual boson. All plots are taken from [27].

decrease slightly when going to NLO. For $v$, this effect is less pronounced than for the LHC case, which may be attributed to a much stronger contribution by the $q \bar{q}$ channel in the present $p \bar{p}$ case, which receives smaller radiative corrections. Again, this feature was predicted phenomenologically in Ref. [30].

We now consider the fixed-target regime, where we start with a comparison to the Fermilab E866/NuSea data taken with an $800 \mathrm{GeV}$ proton beam in $p p$ [9] and $p d$ [8] scattering. The comparisons to the two data sets are shown in Figs. $2 \mathrm{a}$ and $2 \mathrm{~b}$. We first note that the $p p$ data are overall in much better agreement with the theoretical curves than the $p d$ ones. For $p p$ scattering, the coefficient $\lambda$ is well described, given the relatively large experimental uncertainties. There is a slight trend in the data for the coefficient $v$ to be lower than the theoretical prediction. The NLO 

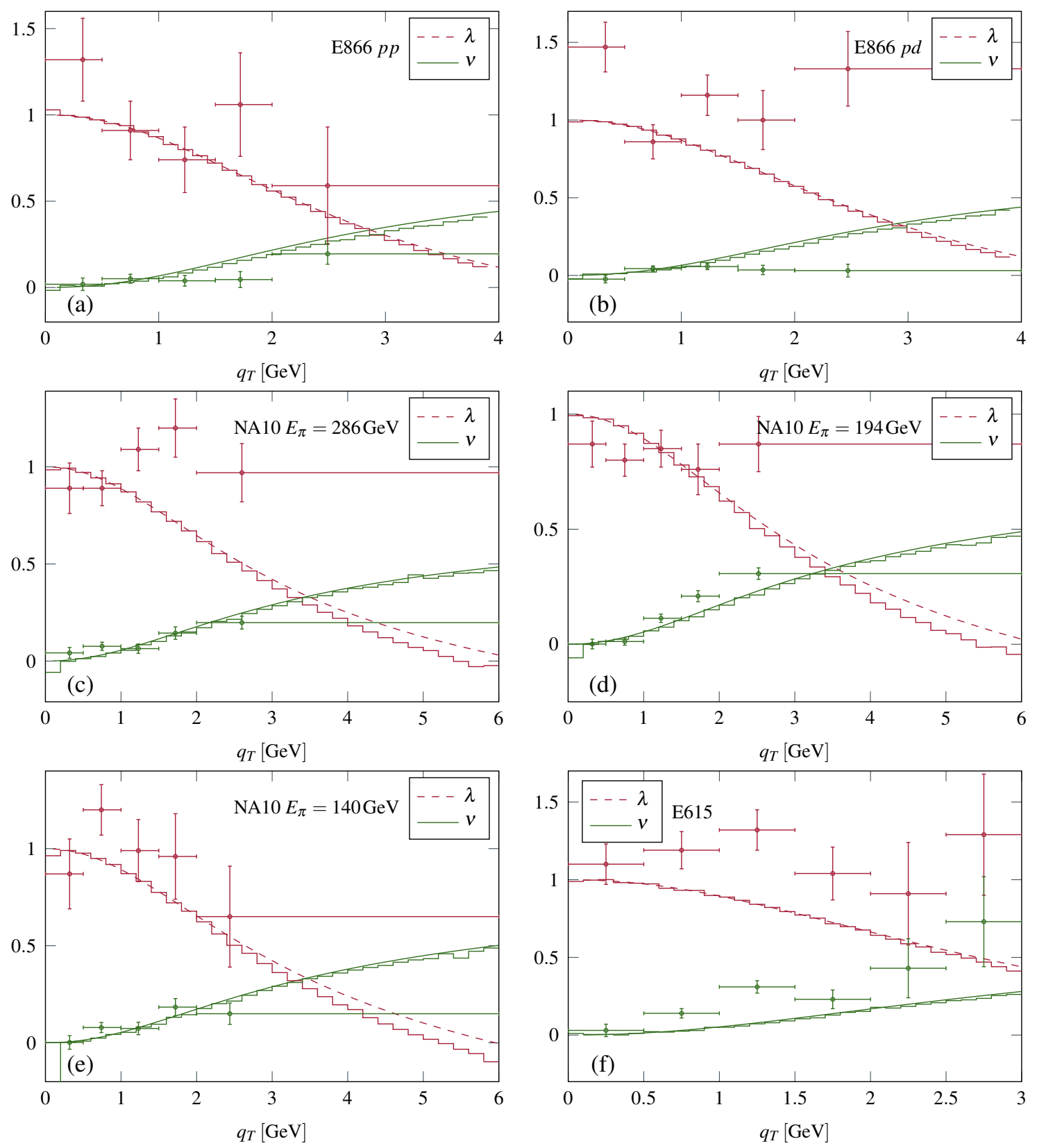

Figure 2: Comparison of LO (lines) and NLO (FEWZ [28], histograms) theoretical results to E866 [9, 8] ((a) and (b)), NA10 [6] ((c), (d) and (e)) and E615 [7] (f) data. E866 data correspond to $p p$ and $p d$ scattering respectively, taken with an $800 \mathrm{GeV}$ beam. Error bars are statistical only. We have integrated over the mass range $4.5 \leq Q \leq 15 \mathrm{GeV}$, excluding the bottomonium region $9 \leq Q \leq$ $10.7 \mathrm{GeV}$, and over $0 \leq x_{F} \leq 0.8$. NA10 data are taken with pion beam energy $E_{\pi}=286 \mathrm{GeV}, E_{\pi}=$ $194 \mathrm{GeV}$ and $E_{\pi}=140 \mathrm{GeV}$ respectively. Error bars are statistical only. We have integrated over the mass range $Q \geq 4 \mathrm{GeV}\left(Q \geq 4.05 \mathrm{GeV}\right.$ for $\left.E_{\pi}=194 \mathrm{GeV}\right)$, excluding the bottomonium region $8.5 \leq$ $Q \leq 11 \mathrm{GeV}$, and implemented the cut $0 \leq x_{\pi} \leq 0.7$. E615 data are taken with pion beam energy $E_{\pi}=252 \mathrm{GeV}$. We have integrated over the mass range $4.05 \leq Q \leq 8.55 \mathrm{GeV}$ and implemented the cuts $0 \leq x_{F} \leq 1$ and $0.2 \leq x_{\pi} \leq 1 . x_{F}=2 q_{L} / \sqrt{s}$ is the Feynman variable and is counted as positive in the forward direction of the beam. $x_{\pi}$ is defined by $x_{\pi}=\frac{1}{2}\left(x_{F}+\sqrt{x_{F}^{2}+4 Q^{2} / s}\right)$. Taken from [27]. 
corrections in fact provide a slight improvement here. For $p d$ scattering, the two data points for $v$ at the highest $q_{T}$ are clearly below theory even at NLO. The coefficient $\lambda$ is not well described, neither at LO nor at NLO. An important point to note in this context is the positivity constraint [2] $W_{L} \geq 0$, which immediately implies

$$
\lambda \leq 1
$$

This condition is completely general and relies only on the hermiticity of the neutral current. It is interesting to observe that the $p d$ data are only in borderline agreement with this constraint.

Going further down in energy, we finally discuss the data from the $\pi+$ tungsten scattering experiments NA10 [6] and E615 [7]. NA10 used three different energies for the incident pions, $E_{\pi}=286,194,140 \mathrm{GeV}$, while E615 operated a pion beam with energy $252 \mathrm{GeV}$. Figures 2c, 2d and 2e show the comparisons of our LO and NLO results for $\lambda$ and $v$ to the NA10 data. The NLO corrections are overall small for $v$, but for $\lambda$ they become more pronounced toward larger $q_{T}$. We note that NLO results for one of the NA10 energies were also reported in Ref. [32], where however not the appropriate kinematical regime in $Q$ was chosen, leading to an underestimate of $v$ which has unfortunately given rise to the general notion in the literature that perturbative QCD cannot describe the Drell-Yan angular coefficients. We also note that for the kinematics used in [32] the NLO corrections appear to be somewhat smaller than the ones we find here. The three cases shown in Figs. 2c, 2d and 2e have in common that the data for $v$ are well described, perhaps slightly less so for the pion energy $194 \mathrm{GeV}$. The experimental uncertainties for the coefficient $\lambda$ are very large, and it is not possible to draw solid conclusions from the comparison. We note that wherever there are tensions between data and theory concerning $\lambda$, the data tend to lie uncomfortably close to (or even above) the positivity constraint $\lambda \leq 1$.

In case of E615, we find the results shown in Fig. 2f. We observe that neither the description of $\lambda$ nor that of $v$ is good. The NLO corrections are overall small and thus do not change this picture. It is clear that on the basis of the data one would derive a significant violation of the Lam-Tung relation (1.5), since $\lambda$ and $v$ both enter the relation with the same sign, and the data for both $\lambda$ and $v$ are higher than theory (the latter satisfying the relation at LO). It is worth pointing out, however, that the experimental uncertainties are large and, more importantly, again the data show a certain tension with respect to the positivity limit (2.1).

\section{Recent developments}

In summer 2016 the ATLAS Collaboration published new data on the angular coefficients at $\sqrt{s}=8 \mathrm{TeV}$ for Z-Boson events [12]. The uncertainties quoted by ATLAS are overall much smaller than the CMS ones. The results are shown in figure 3. For the coefficient $\lambda$ the data confirm theory both in leading and in next-to-leading order which show little difference. This is different, however, for the coefficient $v$. Unlike for the CMS data, even NLO fails to describe the data well, as is evident from figures $3 \mathrm{a}, 3 \mathrm{~b}$ and $3 \mathrm{c}$. The agreement is reasonable for the extremely forward/backward rapidity bin, where however the experimental uncertainties are large.

The tension between theory and data observed in the figures requires further attention. It appears unlikely that effects related to the logarithms in $q_{T} / Q$ mentioned above or to transversemomentum dependent parton distributions would have any impact on the predicted $v$ at transverse 
momenta of the order of a few tens of GeV or even above. If the data persist, NNLO effects would arguably offer the only viable explanation for the observed trend. Fortunately, NNLO predictions are expected to become available very soon [35]. In the meantime, we investigate some features of the NLO results. Figure 3 e shows the " $K$-factors" relevant for the coefficient $v$, that is,

$$
K_{T L} \equiv \frac{\left(W_{T}+W_{L}\right)_{\mathrm{NLO}}}{\left(W_{T}+W_{L}\right)_{\mathrm{LO}}}, \quad K_{\Delta \Delta} \equiv \frac{\left(W_{\Delta \Delta}\right)_{\mathrm{NLO}}}{\left(W_{\Delta \Delta}\right)_{\mathrm{LO}}} .
$$

As one can see, the $K$-factor is much bigger for the denominator of $v$ than for the numerator. If this trend continues at the next order, a smaller $v$ will result, which will be in better agreement with the data. We note, however, that the additional NNLO contributions would have to be quite large in order to lead to a good description of the data.

Figure $3 \mathrm{f}$ shows the different channels contributing to the numerator of $v$. We distinguish among the $g g, q g$, and $q \bar{q}+q q$ subprocesses, where in the latter case all possible flavor contributions are included. It is straightforward to extract the subprocess contributions by suitably setting the quark or gluon PDFs to zero. We always keep all channels in the denominator of $v$. As one can see, when going from LO to NLO all channels receive corrections that drive the result closer towards the data. The $q g$ channel is the major contributor and decreases considerably at NLO. The $q \bar{q}+q q$ contribution becomes smaller in the $q_{T}$ regime of interest, despite the fact that a lot of more initial states are possible at NLO. Finally the $g g$ channel, which is new at NLO, contributes negatively, lowering the overall $v$.

\section{Conclusions}

We have presented detailed and exhaustive comparisons of data for the Drell-Yan lepton angular coefficients $\lambda$ and $v$ to LO and NLO perturbative-QCD calculations. To obtain NLO results, we have employed public codes that allow us to compute the full Drell-Yan cross section at NNLO, and in which the angular pieces we are interested in are contained.

Our numerical results show that overall perturbative QCD is able to describe the experimental data quite well, except for the new ATLAS data. For the CMS data the agreement is very good when the NLO corrections are taken into account. This finding is in line with arguments made in the recent literature [30]. Also the Tevatron data are very well described at NLO. Toward the fixedtarget regime, we again find an overall good agreement, with possible exceptions for the E866 pd data set for $v$ at high $q_{T}$ and for the E615 data. We remark that the latter data set carries large uncertainties and also hints at tensions with the positivity constraint $\lambda \leq 1$.

To be sure, the description of the cross sections that enter the angular coefficients requires input beyond fixed-order QCD perturbation theory, notably in terms of resummations of logarithms in $q_{T} / Q$ and of transverse-momentum dependent parton distributions. On the other hand, based on the angular coefficients alone, in our view there is no convincing evidence for any effects other than the ones we have considered here. In particular, we argue that one should dispel the myth that perturbative QCD is not able to describe the Drell-Yan angular coefficients, which in fact has been iterated over and over in the literature. While we most certainly do not wish to exclude the presence of contributions by the Boer-Mulders effect in the $\cos 2 \phi$ part of the angular distribution, it is also clear from our study that future phenomenological studies of the effect should incorporate the QCD radiative effects. 

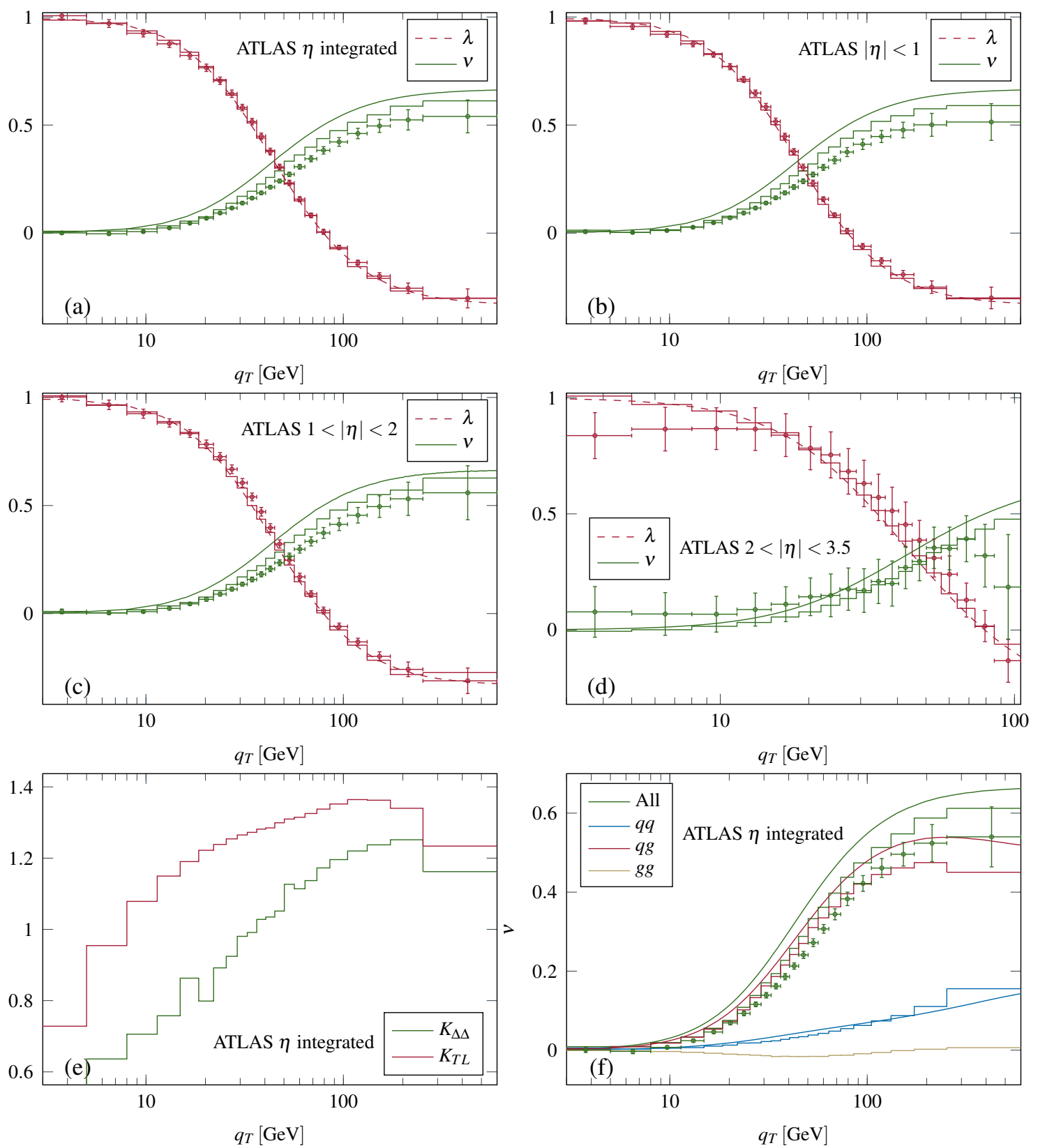

Figure 3: (a)-(d) show the comparison of LO (lines) and NLO (FEWZ [28], histograms) theoretical results for the angular coefficients $\lambda$ and $v$ to the ATLAS data [12]. Data are taken at $\sqrt{s}=$ $8 \mathrm{TeV}$, integrated over $80 \leq Q \leq 100 \mathrm{GeV}$ and over the full rapidity interval, a mid rapidity interval $|\eta|<1$, a forward/backward rapidity interval $1<|\eta|<2$ and an extremely forward/backward rapidity interval $2<|\eta|<3.5$ of the virtual boson, respectively. In (e) the $K$-factors defined in equation (3.1) are shown. (f) illustrates the contributions by the different partonic channels. 
It will be interesting to see whether the gap between theory and the ATLAS data will be bridged by the NNLO contributions. Concerning the fixed-target regime, our results clearly make the case for additional new precision data for the Drell-Yan angular coefficients that would allow to convincingly establish whether there are departures from the "plain" QCD radiative effects we have considered here. We hope that such data will be forthcoming from measurements at the COMPASS [33] or E906 [34] experiments, or possibly at RHIC.

\section{References}

[1] J. C. Collins and D. E. Soper, Phys. Rev. D 16, 2219 (1977).

[2] C. S. Lam and W. K. Tung, Phys. Rev. D 18, 2447 (1978).

[3] D. Boer and W. Vogelsang, Phys. Rev. D 74, 014004 (2006) [hep-ph/0604177].

[4] E. L. Berger, J. W. Qiu and R. A. Rodriguez-Pedraza, Phys. Lett. B 656, 74 (2007) [arXiv:0707.3150 [hep-ph]]; Phys. Rev. D 76, 074006 (2007) [arXiv:0708.0578 [hep-ph]].

[5] For review, see J. C. Peng and J. W. Qiu, Prog. Part. Nucl. Phys. 76, 43 (2014) [arXiv:1401.0934 [hep-ph]].

[6] M. Guanziroli et al. (NA10 Collaboration), Z. Phys. C 37, 545 (1988).

[7] J. S. Conway et al., Phys. Rev. D 39, 92 (1989).

[8] L. Y. Zhu et al. (FNAL E866/NuSea Collaboration), Phys. Rev. Lett. 99, 082301 (2007) [hep-ex/0609005].

[9] L. Y. Zhu et al. (FNAL E866/NuSea Collaboration), Phys. Rev. Lett. 102, 182001 (2009) [arXiv:0811.4589 [nucl-ex]].

[10] T. Aaltonen et al. (CDF Collaboration), Phys. Rev. Lett. 106, 241801 (2011) [arXiv:1103.5699 [hep-ex]].

[11] V. Khachatryan et al. (CMS Collaboration), Phys. Lett. B 750, 154 (2015) [arXiv:1504.03512 [hep-ex]].

[12] G. Aad et al. [ATLAS Collaboration], JHEP 1608, 159 (2016) [arXiv:1606.00689 [hep-ex]].

[13] D. Boer, Phys. Rev. D 60, 014012 (1999) [hep-ph/9902255].

[14] D. Boer and P. J. Mulders, Phys. Rev. D 57, 5780 (1998) [hep-ph/9711485].

[15] S. J. Brodsky, D. S. Hwang and I. Schmidt, Phys. Lett. B 530, 99 (2002) [hep-ph/0201296]; Nucl. Phys. B 642, 344 (2002) [hep-ph/0206259]; J. C. Collins, Phys. Lett. B 536, 43 (2002) [hep-ph/0204004].

[16] V. Barone, S. Melis and A. Prokudin, Phys. Rev. D 82, 114025 (2010) [arXiv:1009.3423 [hep-ph]].

[17] Z. Lu and I. Schmidt, Phys. Rev. D 84, 094002 (2011) [arXiv:1107.4693 [hep-ph]].

[18] D. Boer, S. J. Brodsky and D. S. Hwang, Phys. Rev. D 67, 054003 (2003) [hep-ph/0211110]; Z. Lu and B. Q. Ma, Phys. Lett. B 615, 200 (2005) [hep-ph/0504184]; B. Pasquini and P. Schweitzer, Phys. Rev. D 90, 014050 (2014) [arXiv:1406.2056 [hep-ph]].

[19] C. S. Lam and W. K. Tung, Phys. Lett. B 80, 228 (1979).

[20] J. C. Collins, Phys. Rev. Lett. 42, 291 (1979). 
[21] K. Kajantie, J. Lindfors and R. Raitio, Phys. Lett. B 74, 384 (1978).

[22] J. Cleymans and M. Kuroda, Nucl. Phys. B 155, 480 (1979) [Erratum-ibid. B 160, 510 (1979)].

[23] J. Lindfors, Phys. Scr. 20, 19 (1979).

[24] C. S. Lam and W. K. Tung, Phys. Rev. D 21, 2712 (1980).

[25] E. Mirkes, Nucl. Phys. B 387, 3 (1992).

[26] E. Mirkes and J. Ohnemus, Phys. Rev. D 51, 4891 (1995) [hep-ph/9412289].

[27] M. Lambertsen and W. Vogelsang, Phys. Rev. D 93, 114013 (2016) [arXiv:1605.02625 [hep-ph]].

[28] K. Melnikov and F. Petriello, Phys. Rev. D 74, 114017 (2006) [hep-ph/0609070]; R. Gavin, Y. Li, F. Petriello and S. Quackenbush, Comput. Phys. Commun. 182, 2388 (2011) [arXiv:1011.3540 [hep-ph]]; Y. Li and F. Petriello, Phys. Rev. D 86, 094034 (2012) [arXiv:1208.5967 [hep-ph]].

[29] S. Catani, L. Cieri, G. Ferrera, D. de Florian and M. Grazzini, Phys. Rev. Lett. 103, 082001 (2009) [arXiv:0903.2120 [hep-ph]]; S. Catani and M. Grazzini, Phys. Rev. Lett. 98, 222002 (2007) [hep-ph/0703012].

[30] J. C. Peng, W. C. Chang, R. E. McClellan and O. Teryaev, Phys. Lett. B 758, 384 (2016) arXiv:1511.08932 [hep-ph]; J. C. Peng, these proceedings.

[31] A. Bacchetta, D. Boer, M. Diehl and P. J. Mulders, J. High Energy Phys. 08, 023 (2008) [arXiv:0803.0227 [hep-ph]].

[32] A. Brandenburg, O. Nachtmann and E. Mirkes, Z. Phys. C 60, 697 (1993).

[33] M. Chiosso (COMPASS Collaboration), Eur. Phys. J. Web Conf. 85, 02036 (2015).

[34] K. Nakano (E906/SeaQuest Collaboration), Int. J. Mod. Phys. Conf. Ser. 40, 1660041 (2016).

[35] A. Gehrmann-De Ridder, T. Gehrmann, E. W. N. Glover, A. Huss and T. A. Morgan, JHEP 1607, 133 (2016) [arXiv:1605.04295 [hep-ph]]; JHEP 1611, 094 (2016) [arXiv:1610.01843 [hep-ph]]. 